\section{EXCISION OF TONSILS.}

Read before the Indiana State Medical Association, May, 1898.

BY W. H. PETERS, B.S., M.D.

LAFAYETTE, IND.

As to the anatomy and pathology of the tonsils, I will only say that the normal throat has nothing of the kind in it. And as to treatment, nothing but removal of the diseased glandular mass gives the desired relief.

Many plans are in use to accomplish this. The guillotine will slice off the tonsil, and in children, if the claws which complicate it are removed and suitable forceps used in their place, the guillotine is fairly satisfactory, but hemorrhage is free. Though not dangerous in children, this hemorrhage is troublesome, because it interferes with the simultaneous removal of the pharyngeal tonsil, which is almost invariably present. In the adult, however, this hemorrhage is a serious matter, and has been fatal in many cases. To avoid this Burnett and other authors recommend that a snare be used-the growth being cut through gradually--several hours being consumed in the operation. The method is worthy of the Inquisition. The galvanocautery loop I must condemn by using Burnett's own words: "We may resort to the cautery in suitable cases, which are certainly exceptional, with a feeling of security and with the expec. tation of getting a thorough and satisfactory result." My personal experience with the cautery loop has been confined to a single case, in which acute otitis media in both ears resulted on the fourth day. Other authors have devised an array of scissors, cutting forceps and knives which do the work well, but the oper. ation is a bloody one, requiring general anesthesia and an hour's time to complete it. Ignipuncture is too tedious, months being required and a bad cicatricial stump remaining.

The guillotine, then, with its danger of hemorrhage and lack of thoroughness; the cautery, with its limited usefulness; the ordinary snares, with the prolonged torture and shock, certainly indicate a demand for something different. Here let me say that the tonsil must be completely removed; that tonsils which absolutely demand removal are often deeply imbedded or diffused over a surface of an inch and a half by two inches in size. In tonsils of the kind named, the guillotine and cautery snare are useless.

In $1<88$ I devised a tonsil snare which was perfected in 1890, and has been in almost daily use ever since, with such satisfactory results that I beg to present it to you. The instruments I show you belong together. In small children, chloroform is used. In children over 10 years of age and in adults it is unnecessary. In children, the fenestrated tip recommended by Dr Vedder of Pittsburg is always used. The operation is performed as follows:

In infants--the child under chloroform-the patient lies upon a couch, with his head toward an artificial light four feet away; my assistant kneels at the patient's head and I stoop over the patient at his right side. The instrument has been prepared as you see --two extra fenestrated tips being held in reserve. Here let me say that the screw adjustment in this instrument is never under any circumstances used, except to regulate the size of the loop before operating. The patient's tongue is held down by myself or my assistant, as the case may require. If the tonsil is prominent, no forceps are used, and I hold the tongue-depressor myself. If not prominent, my assistant holds the tongue, the forceps are passed through the loop, the tonsil seized, traction made, and with a single stroke the tonsil severed. The other tonsil is removed at the same time. The operation up to this time is bloodless, and now the finger explores the nasopharynx for adenoid trouble. If present, and it usually is, the mass is removed by Gottstein's curette. This operation is attended by free hemorrhage, and rouses the child. The whole operation has taken less than a minute, and the child is rolled to the edge of the couch, with his face over the opening of a fountain cuspidor. (This latter is of my own design. It takes care of any vomited matters, etc, though vomiting is very rare.) In adults a 25 per cent. solution of cocain is applied around the tonsil and in its crypts, and the tonsil is seized, drawn through the loop and severed by a single stroke.

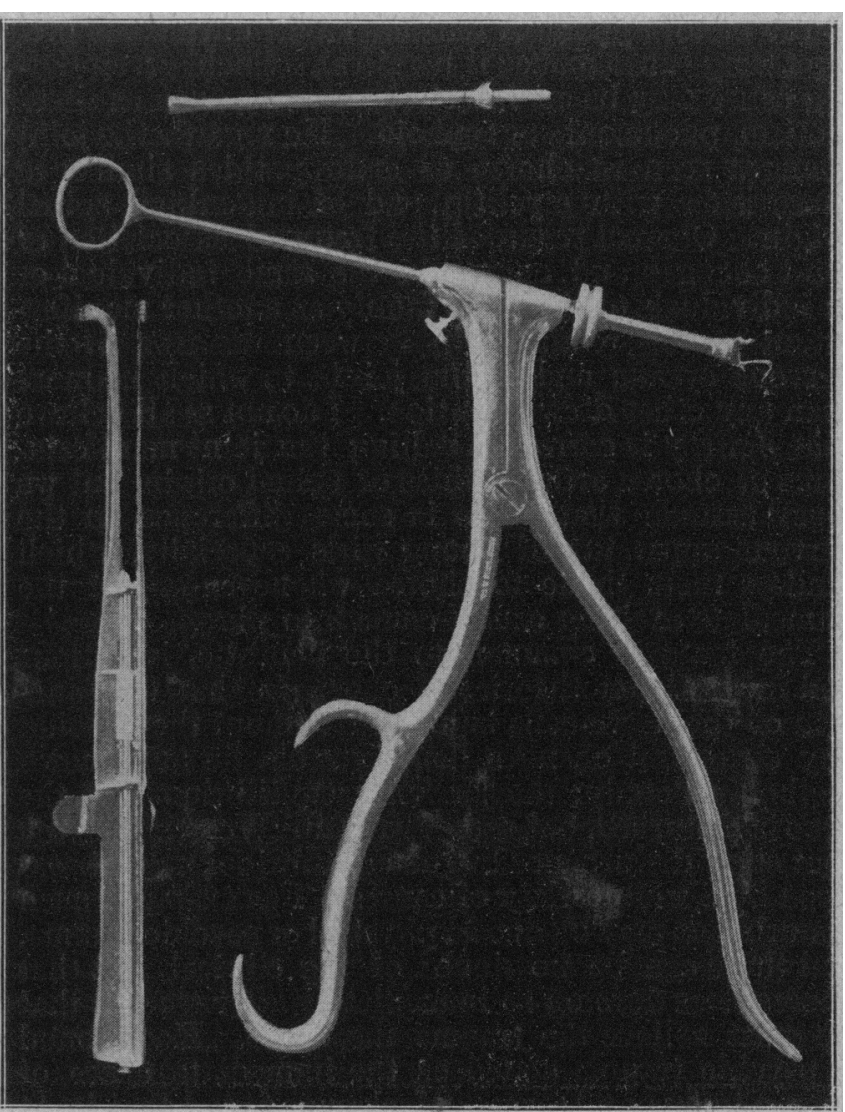

In some tonsils, one in six, perhaps, some dissect ting is necessary, and this requires only two or three rapid strokes of the knife. When no chloroform is used the patient himself, even among children, holds the tongue down with a tongue-depressor while the operation is being performed. In many cases of diffused tonsils a second or third application of the snare, without the patient leaving the chair, is necessary.

As a rule, the hemorrhage does not exceed a few drops. Only in one case has it been sharp--that of Miss Hattie K, 14 years old, living at Rensselaer, Ind., Dr. Berkley being present. In that case the tonsils were enormous, and there was a sharp gush of blood, about two ounces in all, but it stopped kindly, and in no way alarmed the child or rendered me uneasy. She returned a week later and had the second one removed--the same phenomenon recurring. 
I have never seen a drop of secondary hemorrhage from any tonsil.

The snare handle is of machinery or soft steel--and is of the full size shown. A worthless imitation of thie snare is on the market in Chicago. It is too light to be of value. I shall be only too glad to give to any of you drawings and specifications showing proper construction, etc. The forceps have a revolving joint similar to that of the well-known Wecker iris scissors. The forceps are easily aseptible. No. 5 piano steel wire is too small; I use No. 10 . Bosworth's special tonsil snare is a beautiful instrument, but too weak, as it sometimes requires the screw adjustment to complete the operation. The snure I show you will remove any tonsil instantly and easily. The instantaneous écrasement of tonsils by the snare is, I think, original with me, and the instruments certainly are original. I use these instruments in the removal of the lingual tonsils.

Never use a wire the second time. Use a new one. Always twist the ends of the wires securely.

After-treatment.--In children there is little or no pain following and no treatment necessary. In adults, no matter how the tonsils are removed, there will be pain following the operation, except in such individuals as are by nature insensitive. The pain is less in ordinary enlargements where the gland can be wholly removed at a single stroke; but in every case there is a raw surface which will be irritated by swallowing. The patient is ordered to gargle the throat with hot water, to avoid sour and salted foods, and to eat chiefly crackers and milk and medium boiled eggs, for twenty-four hours.

\section{THE TREATMENT OF DISEASES OF PIGMENTATION.}

Read" "Pore the Section on Cutaneous Medicine and"Surgery at the Forty-ninth Annual Meeting of the American Medical Association, held at Denver, Colo., June 7-10, 1898.

BY JOHN V. SHOEMAKER, M.D., LL.D.

PHILADELPHIA.

A clear complexion is one of the elements of personal comelintifs. A very large proportion of people who seek the dermatologist do so on account of some more or less pronounced defect in the normal and healthy coloring of the skin, espacially of the exposed parts. It is needless to say that most of these patients are women. Women are naturally more annoyed and mortified by such blemishes than men. Pigmentary diseases of the skin range from the most trivial to the most serious ailment. Some involve but a slight departure from the normal, others cause extremely dark and unsightly lesions. In most cases the pigmentation is increased or modified in hue, but in a certain number it is diminished. Most diseases in which the pigment of the skin is affected depend upon some more or less grave constitutional cause. A few are merely local disorders.

Lentigo.- This is one of the most common and most insignificant of cutaneous affections. It is purely a local disorder, due to exposure to the heat of the sun and winds of spring and autumn. It is, however, a source of annoyance out of all proportion to its triviality, since it particularly attacks fair and beautiful skins. Freckles consist simply of accumulated masses of normal pigment in the mucous layer of the epiderm. Therefore, it is only necessary to remove this layer in order to cure the disease. The affected parts of the pigment layer may be destroyed by the application of any slightly caustic agent. Among those often used for this purpose are salicylic acid and corrosive sublimate. Touching each spot with strong carbolic acid is a good plan, although a better remedy is copper oleate in the strength of five to sixty grains to the ounce of excipient. The most efficient method of treating these spots is by electricity, used either in the form of electrolysis, inserting the needle superficially over the freckle, or by the frequent application of galvanism.

Chloasma.-Chloasma may be defined as a freckle on a large scale, but, unlike lentigo, it often depends upon the presence of some constitutional disorder. Chlonsma uterinum is mentioned in all the textbooks, and the chloasma of pregnancy sometimes discolors almost the entire body. As regards its external causes, any agency which keeps up a habitual congestion of the skin may give rise to chloasma.

As the discoloration of chloasma resides in the same layer as that of freckles, its local management is exactly the same as in that disease. It is necessary to avoid agents like mustard and cantharides, which themselves are capable of determining pigmentation. The frequent application of the galvanic current is the most efficient means at our disposal for removal of the pigment. External treatment alone will not suffice if the cause is internal. We must address our medication to the constitutional disorder which has produced the chloasma. In many cases we must resort to gynecologic measures. In the chloasma of pregnancy we can make use only of local treatment and those tonic, laxative or diuretic remedies which may be indicated by the general condition of the patient.

Tinea versicolor.-Tinea versicolor or chromophytosis is the disease which in gross appearance most nearly resembles ohloasma. Unlike the latter, it generally spares the face, although it may appear upon the neck and breast. The characteristic lesion of tinea is of a fawn color, very slightly raised above the surrounding surface, and covered with branny scales. The discoloration may be brown or nearly black. The patches of chloasma are not raised and do not scale. Chloasma is not uncommon upon the face. The parasite of tinea may be found by microscopic examination of the scales. The parasite inhabits the horny layer of the epiderm.

The treatment consists in exterminating the microsporon furfur. This object can not be readily accomplished as long as water is freely used upon the parts, as that fluid contributes to the nourishment of the parasite. Parasiticidal agents used in this disease are the mercurial preparations, chrysarobin, sulphurous acid, iodin, turpentin, resorcin, carbolic acid, etc. My favorite remedy in this affection is a 10 to 20 per cent. copper oleate ointment, which insinuates itself between the epidermic cells and destroys the parasite. It is not necessary to use a large quantity. Barely enough should be employed to penetrate the surface, any more than this being a superfluity.

Jaundice.--The discoloration due to absorption of bile pigment varies from light yellow to brown. Its nature should be easily recognized from the stain of the conjunctiva, the discoloration of the urine and its reaction to the bile test, as well as by the accompanying general symptoms. In infants it occurs in conse. quence of the alterations in the circulation taking 